\title{
Unusual quantum confined Stark effect and Aharonov-Bohm oscillations in semiconductor quantum rings with anisotropic effective masses
}

\author{
G. O. de Sousa, ${ }^{1, *}$ D. R. da Costa, ${ }^{1, \dagger}$ Andrey Chaves,${ }^{1,2, \ddagger}$ G. A. Farias,,${ }^{1, \S}$ and F. M. Peeters ${ }^{1,3, \|}$ \\ ${ }^{1}$ Departamento de Física, Universidade Federal do Ceará, Caixa Postal 6030, Campus do Pici, 60455-900 Fortaleza, Ceará, Brazil \\ ${ }^{2}$ Department of Chemistry, Columbia University, 3000 Broadway, New York, New York 10027, USA \\ ${ }^{3}$ Department of Physics, University of Antwerp, Groenenborgerlaan 171, B-2020 Antwerp, Belgium
}

(Received 4 January 2017; revised manuscript received 20 April 2017; published 11 May 2017)

\begin{abstract}
The effects of external electric and magnetic fields on the energy spectrum of quantum rings made out of a bidimensional semiconductor material with anisotropic band structures are investigated within the effective-mass model. The interplay between the effective-mass anisotropy and the radial confinement leads to wave functions that are strongly localized at two diametrically opposite regions where the kinetic energy is lowest due to the highest effective mass. We show that this quantum phenomenon has clear consequences on the behavior of the energy states in the presence of applied in-plane electric fields and out-of-plane magnetic fields. In the former, the quantum confined Stark effect is observed with either linear or quadratic shifts, depending on the direction of the applied field. As for the latter, the usual Aharonov-Bohm oscillations are not observed for a circularly symmetric confining potential, however they can be reinstated if an elliptic ring with an appropriate aspect ratio is chosen.
\end{abstract}

DOI: 10.1103/PhysRevB.95.205414

\section{INTRODUCTION}

The possibility for obtaining atomically thin direct-gap semiconductors from exfoliation of their layered bulk version [1-3] has brought excitement towards the search for different materials that allow for a stable monolayer (or few-layer) form. Density functional theory calculations for some of these materials, namely, black phosphorus (BP) [4], arsenene [5], $\operatorname{ReS}_{2}$ [6], and $\mathrm{TiS}_{3}$ [7], among others, predict anisotropic energy bands which, in some cases, have even been verified experimentally by angle-resolved photoemission spectroscopy [4] and the observation of linear dichroism and polarization-dependent absorption [8-10]. Currently, BP is perhaps the most prominent among the anisotropic twodimensional (2D) materials. It is the most stable crystal form of phosphorus, which has been the subject of renewed interest since its recent isolation and characterization in few layers [4,11-14]. Just like graphene [15,16], silicene [17,18] and germanene $[19,20]$, monolayer black phosphorus (also called phosphorene) is an elemental 2D crystal as opposed to the other materials that compose the layered (van der Waals) materials zoo, such as the transition-metal chalcogenides $[1,3,21,22]$ and boron nitride [23-25]. Phosphorus atoms in phosphorene are organized in a puckered hexagonal lattice, which yields a unique band structure where conductionand valence-band minima are found at the $\Gamma$ point of the Brillouin zone with anisotropic effective masses separated by an energy gap that, depending on the layer number, ranges from $0.3 \mathrm{eV}$ (bulk) to $2.15 \mathrm{eV}$ (monolayer) [11,14,26-31]. Therefore, besides the interesting tunable semiconducting properties of this material, its mass anisotropy, which is reflected in several of its physical properties, such as carrier

\footnotetext{
*gabrieloliveira@ fisica.ufc.br

†diego_rabelo@fisica.ufc.br

†andrey@fisica.ufc.br

§gil@fisica.ufc.br

$\|_{\text {francois.peeters@uantwerpen.be }}$
}

mobility [11], thermal transport [32,33], and even mechanical strain response [34,35], yields the possibility for designing and engineering different electronic devices based on these anisotropic properties $[4,11,36-41]$.

Although a lot is known already about the physics of charge carriers in large flakes of 2D materials, relatively little work has been published on engineered in-plane quantum confined structures in these systems [42]. The local confinement of electrons and holes into finite regions of 2D materials, either by designed electrostatic gates above the layer, or even by just cutting the layer into a desired shape, seems to be a natural step to be taken next in the development of this field. For instance, current technological advances developed for confining carriers in semiconductor-based two-dimensional electron gases into finite regions by gate control [43-45] or by cutting graphene into quantum dots and rings [46-48] could be transferred to the case of general 2D materials, thus enabling the study of quantum confined structures, such as planar semiconductor quantum dots and rings with atomic thickness.

In this paper, we theoretically investigate, within the effective-mass approximation, the energy levels of a 2D semiconductor material with anisotropic effective masses. As a case study, we apply our model to electrons in the conduction band of a few-layer black phosphorus quantum ring. We demonstrate that the effective-mass anisotropy of this system leads to an interesting quantum confinement effect, namely, a localization of the electronic wave function along diametrically opposite regions of the ring. This has consequences, e.g., in the behavior of the confined particle under external applied electric and magnetic fields, as we will demonstrate further on. The physical phenomena discussed here for electrons also are expected to be shared by holes where, e.g., in the case of phosphorene, even a much larger mass anisotropy is present.

\section{THEORETICAL MODEL}

We consider conduction-band electrons in a material with anisotropic mass, confined in a ringlike region defined by 


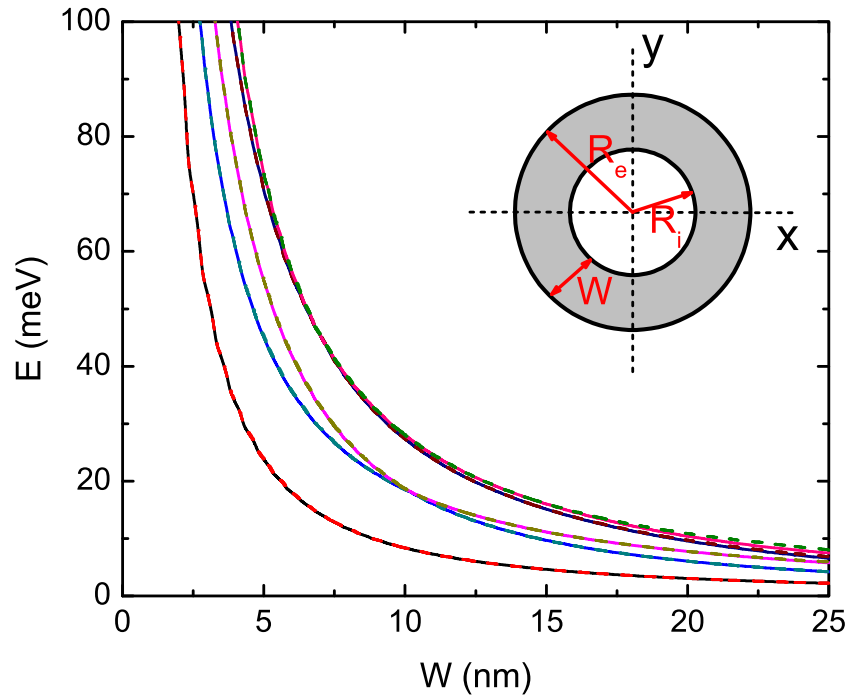

FIG. 1. Ten lowest-energy levels of the BP quantum ring (sketched in the inset) in the absence of applied fields as a function of the ring width ( $W=R_{e}-R_{i}$ ), keeping the average radius fixed as $\left(R_{e}+R_{i}\right) / 2=20 \mathrm{~nm}$.

two circles with internal (external) radius $R_{i(e)}$, as illustrated in the inset of Fig. 1. Within the effective-mass model, the Hamiltonian describing this system is given by

$$
H=\frac{1}{2 m_{0}}\left(\frac{p_{x}^{2}}{m_{x}}+\frac{p_{y}^{2}}{m_{y}}\right)+V(x, y),
$$

where

$$
V(x, y)= \begin{cases}0, & R_{i} \leqslant \sqrt{x^{2}+y^{2}} \leqslant R_{e} \\ \infty, & \text { otherwise. }\end{cases}
$$

Such a confining potential in a gapped 2D material can approximately be achieved experimentally, e.g., by using designed electrostatic gates over the layer [49]. Quantum rings cut out from an infinite layer would also provide infinite potential barriers for electron confinement within the system, but in this case, effects due to zigzag, armchair, or skewed [50] edges would also play a role in the energy spectrum, leading to edge localized states whose energy lies inside the gap [51].

The band-structure anisotropy is reflected in the effective mass, which is different for each in-plane direction. We assume the material parameters for phosphorene, but similar qualitative results are expected for any of the other anisotropic materials mentioned in the Introduction. In fact, the use of such an effective-mass model as described here is justified when we investigate low-energy quasiparticles around a band minimum in a system where bands are sufficiently separate in energy. In the four-band tight-binding model of phosphorene, for example, this would be the case at the energy gap around the $\Gamma$ point: Conduction and valence bands are separate by an $\approx 2 \mathrm{eV}$ gap, whereas the top and bottom bands are separate from them by $\approx 5 \mathrm{eV}$ [39]. In this situation, it is safe to assume electrons populate a single conduction band around $\Gamma$. The electron effective masses in the $x$ and $y$ directions, respectively, are $m_{x}^{e}=0.0826 m_{0}$ and $m_{y}^{e}=1.027 m_{0}$ for monolayer phosphorene [52]. The fact that the effective mass in the $y$ direction is larger than that in the $x$ direction has a direct consequence in the wave-function distributions over the ring as we will demonstrate in what follows. The presence of an in-plane electric field is described by an extra potential $V_{f}(x, y)=e F(x \cos \theta+y \sin , \theta)$ added to Eq. (2), where $\theta$ is the angle that the field makes with the $x$ (armchair) axis. Magnetic fields, on the other hand, are included by the gauge-invariant Peierls-like transformation in the finite difference scheme used to solve Eq. (1) as described by Governale and Ungarelli [53]. In simple words, this transformation consists of writing the finite difference form of the kinetic-energy operators in Eq. (1) as

$$
\begin{aligned}
& K_{i, j}^{(x)}=\frac{\hbar^{2}}{2 m_{0}} \frac{2 \delta_{i j}-U_{x}\left(x_{i}, y_{j}\right) \delta_{i+1, j}-U_{x}^{\dagger}\left(x_{i}, y_{j}\right) \delta_{i-1, j}}{m_{x} \Delta x^{2}}, \\
& K_{i, j}^{(y)}=\frac{\hbar^{2}}{2 m_{0}} \frac{2 \delta_{i j}-U_{y}\left(x_{i}, y_{j}\right) \delta_{i, j+1}-U_{y}^{\dagger}\left(x_{i}, y_{j}\right) \delta_{i, j-1}}{m_{y} \Delta y^{2}},
\end{aligned}
$$

where $\Delta x(y)$ is the grid spacing in the $x(y)$ direction (assumed to be regular over the entire grid) and the phase factor $U_{x(y)}\left(x_{i}, y_{j}\right)=\exp \left[i e \Delta x(y) A_{x(y)}\left(x_{i}, y_{j}\right) / \hbar\right]$ makes the kinetic-energy operator invariant for gauge transformations of the vector potential $\vec{A}$. The Schrödinger equation for the Hamiltonian Eq. (1), discretized in finite differences with the proper transformations to include external magnetic fields, then is solved numerically to obtain the energy levels and wave functions.

\section{STARK EFFECT}

Figure 1 shows the first ten low-lying energy levels of electrons in a BP circular quantum ring as a function of the ring width. As expected, all energy states decrease as the width increases, and they are all doubly degenerate. Surprisingly, even the ground state is doubly degenerate, which is unusual for quantum rings where the ground state is normally a nondegenerate state with angular momentum $l=0$. Throughout the remainder of this paper, internal and external ring radii will be assumed to be $R_{i}=10$ and $R_{e}=20 \mathrm{~nm}$, respectively, unless otherwise stated.

Figure 2 shows the wave functions of the first six lowlying electron states confined in the BP quantum ring. The symmetric/antisymmetric natures of the pairs of states are reminiscent of a double quantum well structure along the $y$ direction, which explains the double degeneracy of the states observed in Fig. 1. In fact, electrons and holes are expected to have their wave functions localized in the upper and lower sides of the ring with an almost zero wave function around $y=0$ : The sectors of the potential around $y \approx 0$ and $y \approx \pm\left(R_{e}+R_{i}\right) / 2$ can be seen as separate quantum wells along the vertical and horizontal directions, respectively. Since the effective mass is larger in the $y$ direction, the confinement energy for a horizontal well is lower, hence the preference of the wave functions for the $y \approx \pm\left(R_{e}+R_{i}\right) / 2$ regions. This is thus a purely quantum-mechanical effect, coming from the nonzero lowest kinetic energy of the confined particles, which is inversely proportional to their effective masses. Another feature that is a consequence of the quantum confinement nature is the increase in the number of nodes in the wave function with higher states. 

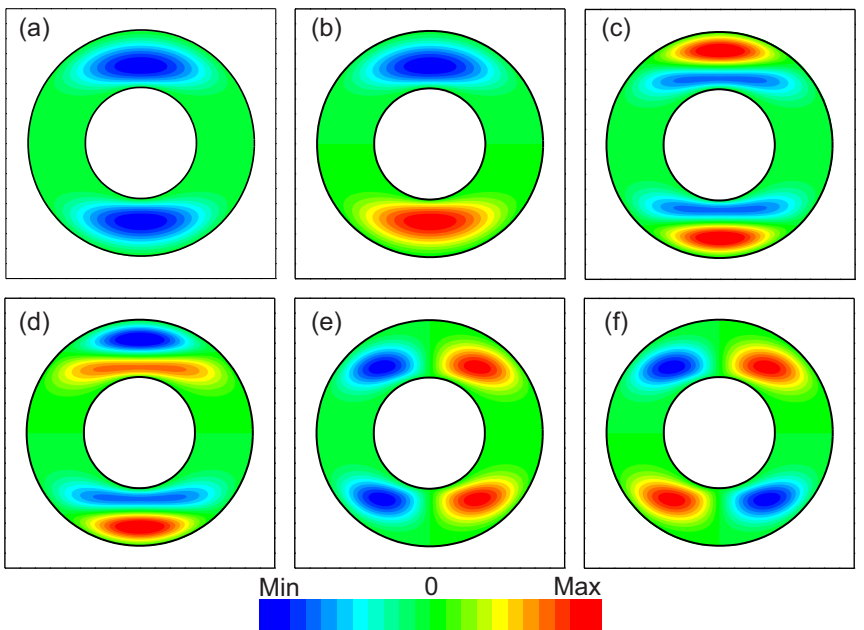

FIG. 2. Wave functions for the first six low-lying states in the absence of electric and magnetic fields, being (a), (c), and (e) symmetric and (b), (d), and (f) antisymmetric. The ring width is fixed as $W=10 \mathrm{~nm}$ with internal (external) radii of $R_{i}=10 \mathrm{~nm}$ $\left(R_{e}=20 \mathrm{~nm}\right)$.

This peculiar double-well-like wave function has interesting consequences on the behavior of the electron and hole energy levels under applied in-plane electric fields. As shown in Fig. 3(a), if the field is applied along the (armchair)

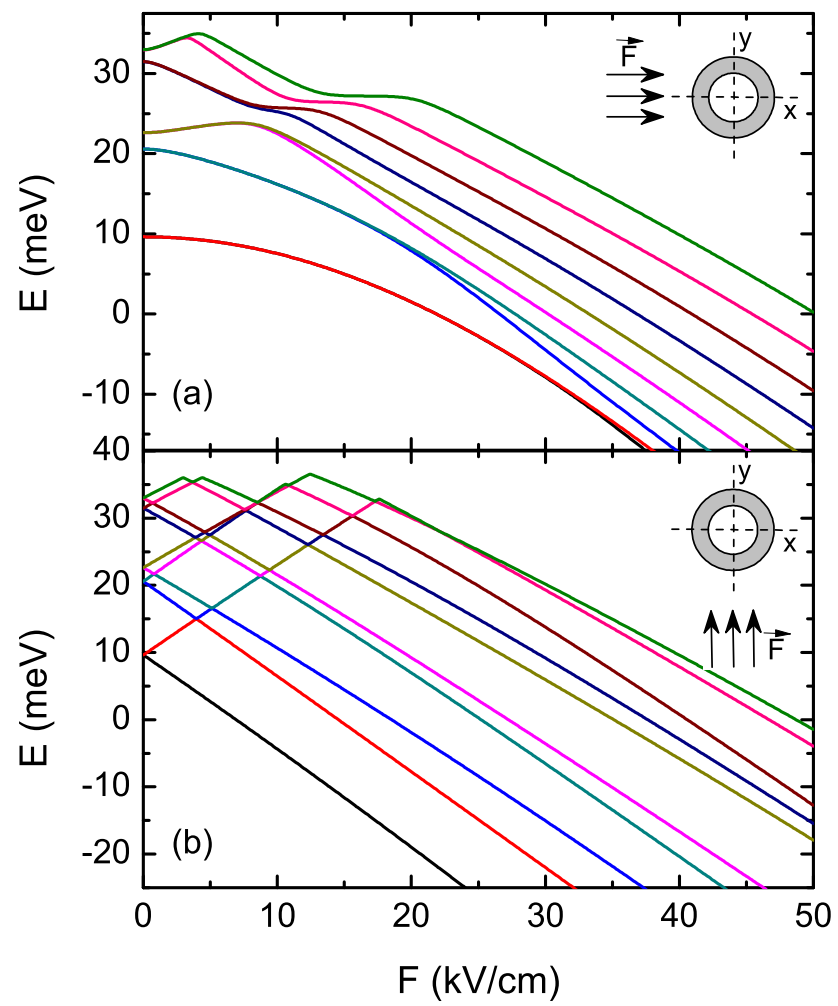

FIG. 3. Lowest ten energy levels of a BP quantum ring as a function of the in-plane electric-field amplitude $F$, being (a) $\theta=0$ and (b) $\theta=\pi / 2$ the angle between the $x$ axis and the vector $\vec{F}$ (see the insets of the figures). The ring width is $W=10 \mathrm{~nm}$ with internal (external) radii of $R_{i}=10 \mathrm{~nm}\left(R_{e}=20 \mathrm{~nm}\right)$.
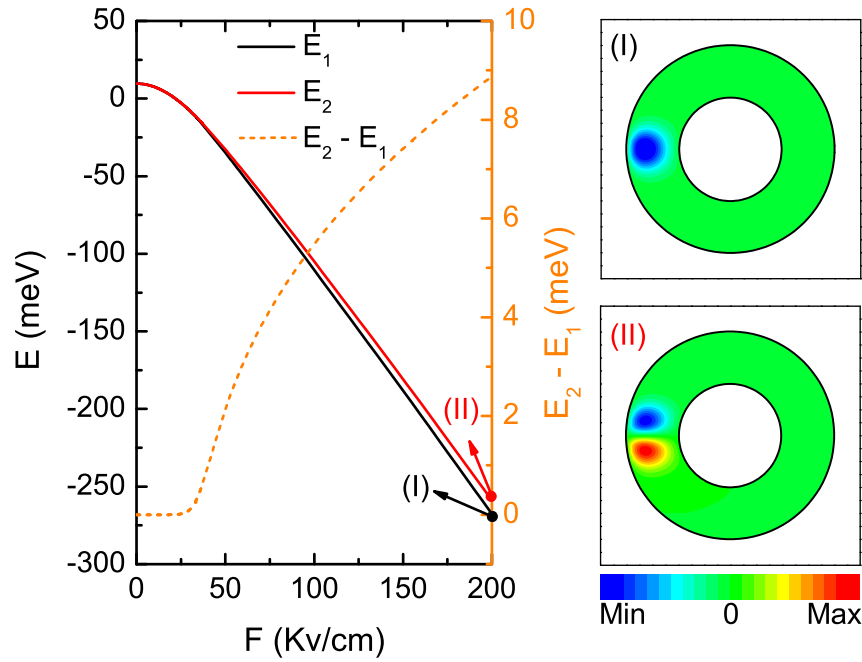

FIG. 4. Splitting $E_{2}-E_{1}$ (right scale) of the first low-lying two energy levels $E_{1}$ and $E_{2}$ (left scale) of a BP quantum ring as a function of the in-plane electric-field amplitude $F$ for $\theta=0$ [see Fig. 3(a)] along with the wave functions for the (I) first and (II) second states at $F=200 \mathrm{kV} / \mathrm{cm}$.

$x$ direction, a regular quadratic quantum confined Stark shift (QCSS) occurs as expected for quantum rings or dots. However, if the field is applied along the (zigzag) $y$ direction, the electric-field dependence of the energies becomes linear with a series of crossings between excited states, thus forming a spectrum that exhibits a close resemblance with the QCSS of a double quantum well [54]. This behavior persists even when considering different values of $W$. In fact, core-shell quantum wires with eccentricity deformations also exhibit a very similar phenomenon [55] where the resemblance is explained as due to the analogy between a situation where the confining potential is elliptic but the kinetic-energy band is a paraboloid (in quantum wires) and a situation where the confining potential is a circle but the kinetic energy is an elliptic paraboloid (in BP quantum rings). This analogy will be discussed in greater detail further on.

The slopes of all curves in Fig. 3(b) for different eigenstates eventually converge to the linear function $E_{n}=-e F\left(R_{e}+\right.$ $\left.R_{i}\right) / 2+E_{n}^{0}$, where $E_{n}^{0}$ is a constant, which is different for each state $n$. This is a consequence of the strong confinement at $y_{t}=-\left(R_{e}+R_{i}\right) / 2$, even for low fields so that the effect of the applied field culminates just in shifting down the electron energy by an amount of $e F y_{t}$.

A more careful analysis of the ground and first excited states in Fig. 3(a) shows that their degeneracy is lifted as the electric field increases. This is illustrated in Fig. 4 where the difference $E_{2}-E_{1}$ significantly increases after $F \approx 30 \mathrm{kV} / \mathrm{cm}$. In fact, higher fields push electron wave functions towards the left part of the ring out of the pockets where they were localized in the absence of fields [see Figs. 2(a) and 2(b)] as one can verify by the color maps of the wave functions for these states, shown in the panels labeled (I) and (II) in Fig. 4. The energy due to a $F \approx 30-\mathrm{kV} / \mathrm{cm}$ field is enough to overcome the kinetic-energy barrier around $y=0$ created by mass anisotropy and, once the electron wave function is no longer in the double-well-like configuration, there is no longer a reason for the ground and 


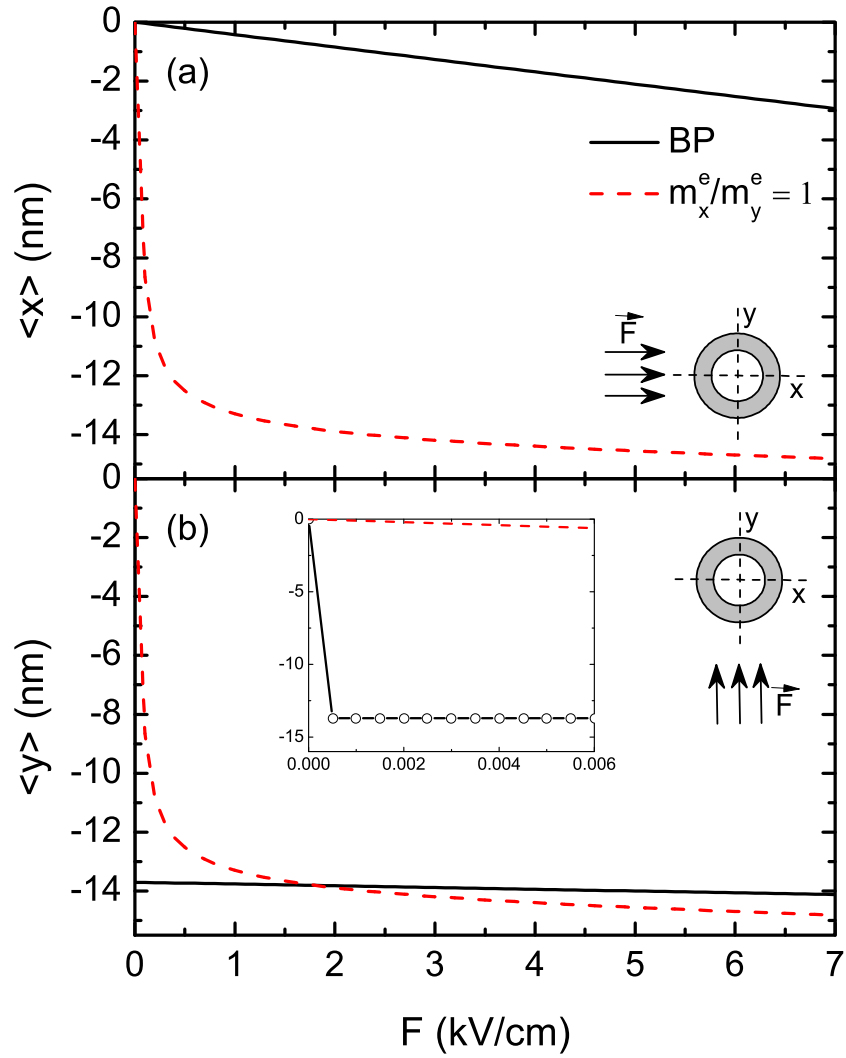

FIG. 5. Average position of the ground state as a function of the in-plane electric-field amplitude $F$ applied in the (a) $x$ and (b) $y$ directions. Two different mass ratios were considered: the one of BP (the solid black line) and $m_{x}^{e} / m_{y}^{e}=1$ (the dashed red line) with $m_{x}^{e}=m_{y}^{e}=1.027 m_{0}$. The inset exhibits a zoom around the low electric-field amplitudes for $F$ pointing towards the $y$ direction, showing that for any field value, the wave function in the $y$ direction moves immediately to such a preferred region inside the quantum ring for the $\mathrm{BP}$ case.

first excited states to be degenerate. On the other hand, the lifting of the degeneracy is very different in Fig. 3(b), and it happens instantaneously when applying the field, that is due to the strong double-well-like confinement.

The average position of the ground-state electron is shown as a function of the external field intensity $F$ by solid lines in Fig. 5. If the field is applied along the positive $x$ direction, Fig. 5(a) shows that $\langle x\rangle$ linearly decreases as expected for circularly confined electrons, such as in atoms and quantum dots. This is however not the behavior found for regular isotropic quantum rings as shown by the red curves for $m_{x}^{e} / m_{y}^{e}=1$ depicted in Figs. 5(a) and 5(b) for comparison, which exhibit a smooth decay. Moreover, if the field points towards the positive $y$ direction, the average position $\langle y\rangle$ abruptly decreases to $\langle y\rangle \approx-14 \mathrm{~nm}$, roughly the average radius of the ring. This is a consequence of the double-well-like structure of this anisotropic mass confinement system where even the weakest applied field immediately makes the lower region of the ring more energetically favorable, thus confining the whole wave function to this region as shown by the inset in Fig. 5(b).

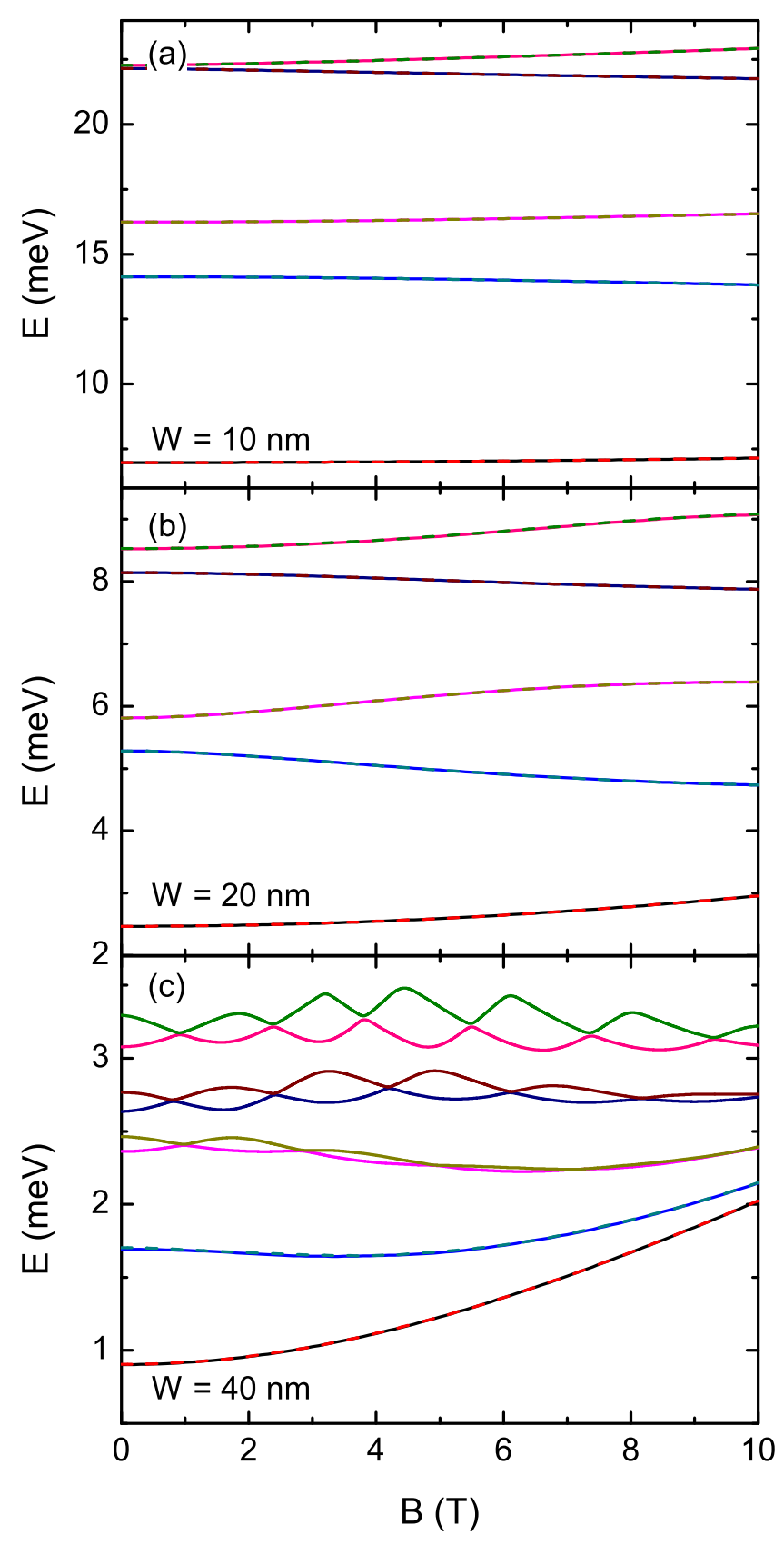

FIG. 6. Energy levels of a circular BP quantum ring as a function of the perpendicular magnetic-field $B$ for different ring widths: (a) $W=10 \mathrm{~nm}$, (b) $W=20 \mathrm{~nm}$, and (c) $W=40 \mathrm{~nm}$. The average radius is kept fixed as $\left(R_{e}+R_{i}\right) / 2=30 \mathrm{~nm}$.

\section{AHARONOV-BOHM OSCILLATIONS}

A ubiquitous feature of semiconductor quantum rings is its adequacy for the study of Aharonov-Bohm (AB) oscillations. It is well known that energy levels of a regular isotropic ring enclosing a magnetic flux $\phi$ oscillate with period $\phi=(n+1 / 2) \phi_{0}$ with an increasing perpendicularly applied magnetic field, where $n=0, \pm 1, \pm 2, \ldots$ is the angular momentum index [56,57]. However, as previously discussed, the mass anisotropy in BP localizes the wave function so that it no longer covers the whole ring and, consequently, $\mathrm{AB}$ oscillations are suppressed as illustrated in Fig. 6 for different 


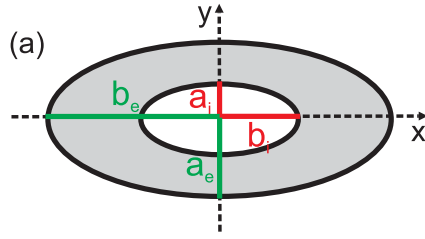

(b)

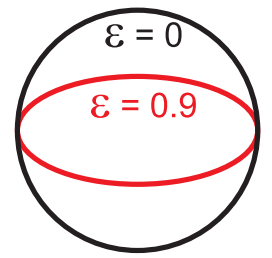

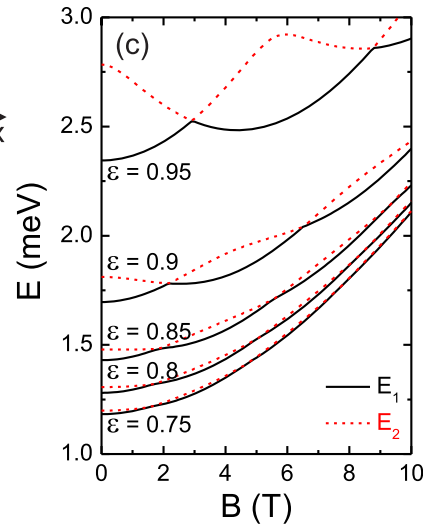

FIG. 7. (a) Elliptical quantum ring potential with (b) two eccentricities: $\varepsilon=0$ (circular) and $\varepsilon=0.9$. (c) The ground (black solid) and the first excited (red dashed) states energies as a function of the external magnetic field, assuming different values of eccentricity, for a fixed ring width of $W=40 \mathrm{~nm}$ and an average radius of $\left(R_{e}+R_{i}\right) / 2=30 \mathrm{~nm}$.

values of ring width. For a ring as wide as $W=40 \mathrm{~nm}$ in Fig. 6(c), AB oscillations still are observed for excited states with higher energy, but the lowest-energy states exhibit no such oscillations and just have their energies increased quadratically with the field (i.e., diamagnetic shift) as in the case of quantum dots in the presence of a magnetic field [58]. Nevertheless, one can bring back the oscillations, even for the ground state, by designing an elliptic quantum ring as sketched in Fig. 7(a) composed of concentric ellipses so that the potential eccentricity "corrects" the eccentricity of the kinetic-energy paraboloid band, coming from mass anisotropy. Figure 7(b) presents a scheme of ellipses with two different eccentricities in order to visualize the effect of high eccentricity. In fact, regions of higher curvature in an elliptic ring are expected to confine particles $[59,60]$, which can thus be used to counterbalance the effective-mass-induced confinement of the circular ring and thus provide more evenly distributed wave functions and, consequently, bring back the $\mathrm{AB}$ oscillations. In this case, the confining potential in Eq. (2) has to be replaced by

$$
V(x, y)= \begin{cases}0, & R_{i} \leqslant \sqrt{x^{2}+\left(y / \sqrt{1-\varepsilon^{2}}\right)^{2}} \leqslant R_{e}, \\ \infty, & \text { otherwise }\end{cases}
$$

where the eccentricity is given by $\varepsilon=1-a_{i} / b_{i}=1-a_{e} / b_{e}$ [ $\varepsilon=0$ in the circular case, see Fig. 7(b)]. $i$ and $e$ denote the inner and outer ellipses, and $a_{i, e}$ and $b_{i, e}$ are the semiminor axis and semimajor axis, respectively [see Fig. 7(a)].

Figure 7(c) shows more pronounced $\mathrm{AB}$ oscillations in the ground and first excited states as a function of a magnetic field with increasing eccentricity. Figure 8 shows that, in the elliptical $(\varepsilon=0.9)$ confinement case, as the width increases, the wave function spreads out more over the ring. The lowest-energy levels in Fig. 8(a) are almost independent of the magnetic field, but, as the ring is made wider, the energy levels exhibit the $\mathrm{AB}$ oscillatory feature [see Figs. 8(b) and 8(c)]. Nevertheless, the mass anisotropy effect is stronger, and the wave function (insets) still keeps its two peaks so that the energy levels group into twofold oscillating sub-bands. States

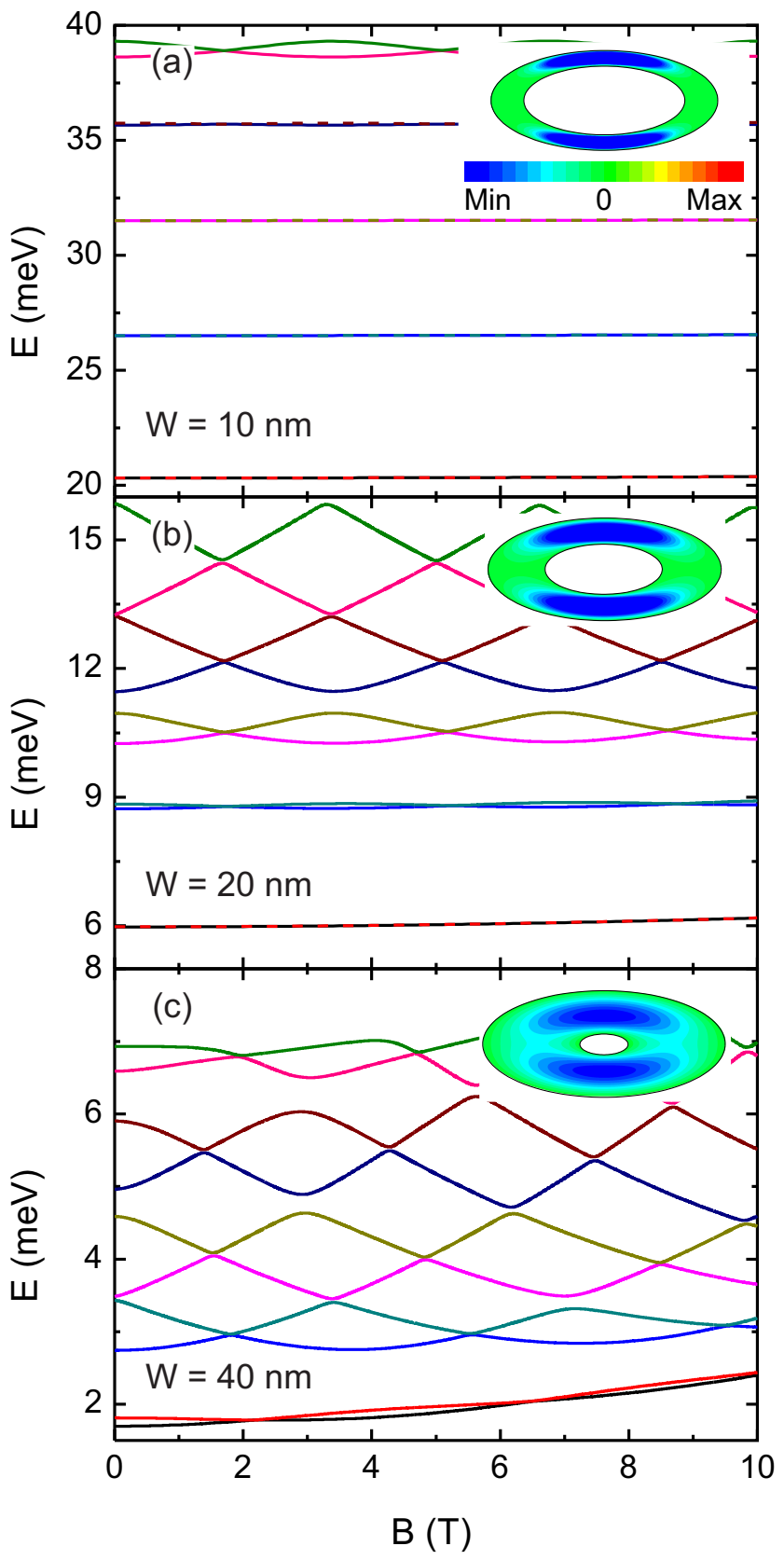

FIG. 8. Energy levels of an elliptic $(\varepsilon=0.9)$ BP quantum ring as a function of perpendicular magnetic-field $B$ for ring widths (a) $W=$ $10 \mathrm{~nm}$, (b) $W=20 \mathrm{~nm}$, and (c) $W=40 \mathrm{~nm}$. The average radius is kept fixed at $\left(R_{e}+R_{i}\right) / 2=30 \mathrm{~nm}$. The insets show the ground-state wave functions for the three different widths and $B=0 \mathrm{~T}$. The red (blue) color represents high (low) wave-function amplitude.

in the same sub-band exhibit periodic crossings, whereas different sub-bands are separated by anticrossings. Notice that the period of $\mathrm{AB}$ oscillations in Fig. 8 is slightly different for each ring width as a consequence of the different areas enclosed by rings with different widths. The same is true for rings with different eccentricities in Fig. 7(c). The minima of the ground-state oscillations in Fig. 8(c) rise in energy as the magnetic field increases (black line). This is expected since, as the magnetic field increases, the magnetic length is reduced, squeezing the electron towards the central region 
and, consequently, increasing its energy. Notice the width of the circular ring in Fig. 6(b) is the same as the one for the elliptic ring in Fig. 8(b). Yet, only the latter exhibits clear $\mathrm{AB}$ oscillations, especially for excited states, thus proving the importance of the ring eccentricity in observing the AB effect in quantum rings made out of BP.

\section{CONCLUSIONS}

We have investigated the effect of applied electric and magnetic fields on the energy levels of conduction-band electrons confined into a quantum ring made of an anisotropic material. Due to the mass anisotropy, the probability density is demonstrated to be not evenly distributed along the ring but rather concentrated in two diametrically opposite regions of the system. As a consequence, our results show that, if the applied electric field points towards the armchair direction, the energy-level and wave-function behaviors are reminiscent of that of a pair of stacked (independent) quantum dots (quadratic Stark shift), whereas if the same field points towards the zigzag direction, the system behaves as coupled quantum wells (linear Stark shift). This is related to the higher effective mass in the $y$ direction (zigzag direction). Thus, this feature can be used, e.g., to analyze the crystal directions of the system.

Furthermore, in the presence of a perpendicular magnetic field, our results also show that a circular anisotropic mass ring does not exhibit $\mathrm{AB}$ oscillations in the energy spectrum, which also is attributed to the anisotropy-induced wave-function localization. However, if the electron is confined rather by an elliptical potential ring, the localization effect is reduced, and it becomes possible to recover the $\mathrm{AB}$ oscillations.

Our numerical results were obtained using parameters for monolayer black phosphorus, although the same qualitative features are expected for any system with effective-mass anisotropy, such as arsenene $\mathrm{ReS}_{2}$ and even multilayer black phosphorus.

\section{ACKNOWLEDGMENTS}

This work was financially supported by $\mathrm{CNPq}$ under the PRONEX/FUNCAP grants, CAPES Foundation, the Flemish Science Foundation (FWO-Vl), and the Brazilian Program Science Without Borders (CsF).
[1] K. F. Mak, C. Lee, J. Hone, J. Shan, and T. F. Heinz, Phys. Rev. Lett. 105, 136805 (2010).

[2] T. Low, A. Chaves, J. D. Caldwell, A. Kumar, N. X. Fang, P. Avouris, T. F. Heinz, F. Guinea, L. Martin-Moreno, and F. Koppens, Nat. Mater. 16, 182 (2017).

[3] V. Sorkin, H. Pan, H. Shi, S. Y. Quek, and Y. W. Zhang, Crit. Rev. Solid State Mater. Sci. 39, 319 (2014).

[4] L. Li, Y. Yu, G. J. Ye, Q. Ge, X. Ou, H. Wu, D. Feng, X. H. Chen, and Y. Zhang, Nat. Nanotechnol. 9, 372 (2014).

[5] C. Kamal and M. Ezawa, Phys. Rev. B 91, 085423 (2015).

[6] H.-X. Zhong, S. Gao, J.-J. Shi, and L. Yang, Phys. Rev. B 92, 115438 (2015).

[7] J. Dai and X. C. Zeng, Angew. Chem., Int. Ed. 54, 7572 (2015).

[8] J. Qiao, X. Kong, Z.-X. Hu, F. Yang, and W. Ji, Nat. Commun. 5, 4475 (2014).

[9] G. Zhang, S. Huang, A. Chaves, C. Song, V. Ongun Özçelik, T. Low, and H. Yan, Nat. Commun. 8, 14071 (2017).

[10] O. B. Aslan, D. A. Chenet, A. M. van der Zande, J. C. Hone, and Tony F. Heinz, ACS Photon. 3, 96 (2016).

[11] H. Liu, A. T. Neal, Z. Zhu, Z. Luo, X. Xu, D. Tománek, and P. D. Ye, ACS Nano 8, 4033 (2014).

[12] F. Xia, H. Wang, and Y. Jia, Nat. Commun. 5, 4458 (2014).

[13] S. P. Koenig, R. A. Doganov, H. Schmidt, A. H. Castro Neto, and B. Özyilmaz, Appl. Phys. Lett. 104, 103106 (2014).

[14] A. Castellanos-Gomez, L. Vicarelli, E. Prada, J. O. Island, K. L. Narasimha-Acharya, S. I. Blanter, D. J. Groenendijk, M. Buscema, G. A. Steele, J. V. Alvarez, H. W. Zandbergen, J. J. Palacios, and H. S. J. van der Zant, 2D Mater. 1, 025001 (2014).

[15] K. S. Novoselov, A. K. Geim, S. V. Morozov, D. Jiang, Y. Zhang, S. V. Dubonos, I. V. Grigorieva, and A. A. Firsov, Science 306, 666 (2004).
[16] A. H. Castro Neto, F. Guinea, N. M. R. Peres, K. S. Novoselov, and A. K. Geim, Rev. Mod. Phys. 81, 109 (2009).

[17] J. Zhao, H. Liu, Z. Yu, R. Quhe, S. Zhou, Y. Wang, C. C. Liu, H. Zhong, N. Han, J. Lu, Y. Yao, and K. Wu, Prog. Mater. Sci. 83, 24 (2016).

[18] P. De Padova, C. Ottaviani, C. Quaresima, B. Olivieri, P. Imperatori, E. Salomon, T. Angot, L. Quagliano, C. Romano, A. Vona, M. Muniz-Miranda, A. Generosi, B. Paci, and G. L. Lay, 2D Mater. 1, 021003 (2014).

[19] A. Acun, L. Zhang, P. Bampoulis, M. Farmanbar, A. van Houselt, A. N. Rudenko, M. Lingenfelder, G. Brocks, B. Poelsema, M. I. Katsnelson, and H. J. W. Zandvliet, J. Phys.: Condens. Matter 27, 443002 (2015).

[20] M. E. Dávila, L. Xian, S. Cahangirov, A. Rubio, and G. L. Lay, New J. Phys. 16, 095002 (2014).

[21] B. Radisavljevic, A. Radenovic, J. Brivio, V. Giacometti, and A. Kis, Nat. Nanotechnol. 6, 147 (2011).

[22] Q. H. Wang, K. Kalantar-Zadeh, A. Kis, J. N. Coleman, and M. S. Strano, Nat. Nanotechnol. 7, 699 (2012).

[23] A. Pakdel, C. Zhi, Y. Bando, and D. Golberg, Mater. Today 15, 256 (2012).

[24] S. Carenco, D. Portehault, C. Boissière, N. Mézailles, and C. Sanchez, Chem. Rev. 113, 7981 (2013).

[25] A. Pakdel, Y. Bando, and D. Golberg, Chem. Soc. Rev. 43, 934 (2014).

[26] V. Tran, R. Soklaski, Y. Liang, and L. Yang, Phys. Rev. B 89, 235319 (2014).

[27] A. Castellanos-Gomez, J. Phys. Chem. Lett. 6, 4280 (2015).

[28] K. Dolui and S. Y. Quek, Sci. Rep. 5, 11699 (2015).

[29] S. Das, W. Zhang, M. Demarteau, A. Hoffmann, M. Dubey, and A. Roelofs, Nano Lett. 14, 5733 (2014).

[30] J. Kim, S. S. Baik, S. H. Ryu, Y. Sohn, S. Park, B.-G. Park, J. Denlinger, Y. Yi, H. J. Choi, and K. S. Kim, Science 349, 723 (2015). 
[31] S. Yuan, E. van Veen, M. I. Katsnelson, and R. Roldán, Phys. Rev. B 93, 245433 (2016).

[32] A. Jain and A. J. H. McGaughey, Sci. Rep. 5, 8501 (2015).

[33] T.-H. Liua and C.-C. Chang, Nanoscale 7, 10648 (2015).

[34] A. S. Rodin, A. Carvalho, and A. H. Castro Neto, Phys. Rev. Lett. 112, 176801 (2014).

[35] J.-W. Jiang and H. S. Park, Phys. Rev. B 91, 235118 (2015).

[36] H. Yuan, X. Liu, F. Afshinmanesh, W. Li, G. Xu, J. Sun, B. Lian, A. G. Curto, G. Ye, Y. Hikita, Z. Shen, S.-C. Zhang, X. Chen, M. Brongersma, H. Y. Hwang, and Y. Cui, Nat. Nanotechnol. 10, 707 (2015).

[37] Q. Liu, X. Zhang, L. B. Abdalla, A. Fazzio, and A. Zunger, Nano Lett. 15, 1222 (2015).

[38] E. V. Castro, K. S. Novoselov, S. V. Morozov, N. M. R. Peres, J. M. B. Lopes dos Santos, J. Nilsson, F. Guinea, A. K. Geim, and A. H. Castro Neto, Phys. Rev. Lett. 99, 216802 (2007).

[39] A. N. Rudenko, S. Yuan, and M. I. Katsnelson, Phys. Rev. B 92, 085419 (2015).

[40] P. Delplace, Á. Gómez-León, and G. Platero, Phys. Rev. B 88, 245422 (2013).

[41] C. Dutreix, E. A. Stepanov, and M. I. Katsnelson, Phys. Rev. B 93, 241404(R) (2016).

[42] R. Zhang, X. Y. Zhou, D. Zhang, W. K. Lou, F. Zhai, and K. Chang, 2D Mater. 2, 045012 (2015).

[43] C. Stampfer, J. Güttinger, F. Molitor, D. Graf, T. Ihn, and K. Ensslin, Appl. Phys. Lett. 92, 012102 (2008).

[44] C. Stampfer, E. Schurtenberger, F. Molitor, J. Güttinger, T. Ihn, and K. Ensslin, Nano Lett. 8, 2378 (2008).

[45] J. H. Bardarson, M. Titov, and P. W. Brouwer, Phys. Rev. Lett. 102, 226803 (2009).
[46] M. Zarenia, A. Chaves, G. A. Farias, and F. M. Peeters, Phys. Rev. B 84, 245403 (2011).

[47] D. R. da Costa, A. Chaves, M. Zarenia, J. M. Pereira, Jr., G. A. Farias, and F. M. Peeters, Phys. Rev. B 89, 075418 (2014).

[48] D. A. Bahamon, A. L. C. Pereira, and P. A. Schulz, Phys. Rev. B 79, 125414 (2009).

[49] A. Fuhrer, S. Lüscher, T. Ihn, T. Heinzel, K. Ensslin, W. Wegscheider, and M. Bichler, Nature (London) 413, 822 (2001).

[50] M. M. Grujić, M. Ezawa, M. Ž. Tadić, and F. M. Peeters, Phys. Rev. B 93, 245413 (2016).

[51] D. J. P. de Sousa, L. V. de Castro, D. R. da Costa, and J. M. Pereira, Jr., Phys. Rev. B 94, 235415 (2016).

[52] H. Asahina and A. Morita, J. Phys. C: Solid State Phys. 17, 1839 (1984).

[53] M. Governale and C. Ungarelli, Phys. Rev. B 58, 7816 (1998).

[54] J. M. Llorens, C. Trallero-Giner, A. Garca-Cristbal, and A. Cantarero, Microelectron. J. 33, 355 (2009).

[55] R. Macêdo, J. Costa e Silva, A. Chaves, G. A. Farias, and R. Ferreira, J. Phys.: Condens. Matter 25, 485501 (2013).

[56] S. Viefers, P. Koskinen, P. Singha Deo, and M. Manninen, Physica E 21, 1 (2004).

[57] D. R. da Costa, A. Chaves, W. P. Ferreira, G. A. Farias, and R. Ferreira, J. Phys.: Condens. Matter 29, 165501 (2017).

[58] T. Ihn, Semiconductor Nanostructures: Quantum States and Electronic Transport (Oxford University Press, New York, 2010).

[59] G. A. Farias, M. H. Degani, J. A. K. Freire, J. Costa e Silva, and R. Ferreira, Phys. Rev. B 77, 085316 (2008).

[60] D. Gridin, A. T. I. Adamou, and R. V. Craster, Phys. Rev. B 69, 155317 (2004). 